\title{
Domestic Hotel Management Group's Resources Integrated from Internet Perspective
}

\author{
Jun-yong Lai \\ Department of Electronic Commerce, Shenzhen Tourism College of Jinan University, Guangdong, China
}

\begin{abstract}
This paper analyzes the domestic hotel management group's strong association resources, puts forward a view that as a main body, hotel management group can carry out the resources integration with other departments, and use the integrated marketing communications model to carry on marketing synergy and innovation which based on consumer data. It studies the problems of management structure, system, leader, enterprise culture during the process of integrating data from an Internet thinking and explores the forms of integration, puts forward suggestions for the integration.
\end{abstract}

Keywords - Internet, integration, hotel management group

\section{互联网视角下国内酒店管理集团资源整合营销初探}

\author{
赖俊勇 \\ 暨南大学深圳旅游学院电子商务系, 广东, 中国
}

\begin{abstract}
摘 要 本文分析了国内酒店管理集团的强关联资源, 提出酒店管理集团可以作为主体开展资源整合, 利用整合营销传播模式对 处于资源核心地位的消费者数据进行营销协同与创新。研究互联网视角下对酒店管理集团开展用户为中心的整合可能碰到的管理架构、 体制、企业文化等问题，探索整合的形式，提出整合的建议。
\end{abstract}

关键词 互联网思维, 资源整合, 酒店管理集团

\section{1. 引言}

近年来, 我国的酒店管理集团发展进入一个快通道, 以华住集团、上海锦江国际集团、华侨城酒店管理集团为 代表的旅游饭店集团呈现快速扩张的趋势, 产业集中度不 断提升, 发展势头旺盛。除了高星级酒店以外, 以如家、 七天等为代表的经济连锁酒店通过加盟、集资等方式快速 复制, 迅速扩大市场份额, 成为国内酒店业不可忽视的力 量。然而以洲际酒店集团、喜达屋酒店集团等为代表的国 际知名酒店管理集团在各方面都存在着优势, 它们以深厚 的文化底蕴、悠久的历史沉淀和发达的信息化水平把酒店 管理水平推到一个相当高的高度, 具体表现在网络优势 (全 球订房网络能带来稳定的客源)、品牌优势 (品牌形成强大 的公信力)、规模优势 (有利整体活动推广与费用成本控 制)、集团化优势 (提供一致的服务)、信息化优势 (快速 高效的预订系统、酒店管理系统统一连接)、管理优势 (优 秀的管理文化)、文化优势 (不同的企业文化来细分市场吸
引细分客户群体）等。面对国外酒店业的巨无霸, 国内酒 店管理集团一方面积极学习甚至复制国际酒店管理集团的 管理方式以获得成长和经验, 另一方面也在酛酿着以资金 运作、政策优势、本土化优势等进行弯道超车, 在市场竞 争中获得更加有利的地位。在某种程度上, 这与互联网领 域上众多本土产品和服务击败国外强大对手的案例是具有 共性的, 例如淘宝通过免费策略把 eBay 从市场先行者和统 治者位置拉下, $\mathrm{QQ}$ 利用更符合国人使用习惯的产品实现先 模仿再取代 ICQ, 百度利用政策优势引发搜索引擎市场大 逆转, 小米手机利用粉丝经济与价格优势从三星手中抢得 国内智能手机市场第一的殊荣。互联网思维引入不同领域 都同样会产生颠覆与重生, 酒店业也如是, 与其坐以待变, 不如起而迎之。因此, 有必要从互联网的视角去研究国内 酒店管理集团, 以整合资源, 形成直接连接用户的关系网 络, 迎接必将成为未来消费主力的 80 后、90 后的成长, 在未来的竞争中抢占先机。 


\section{2. 国内酒店管理集团资源分析}

\section{1 国内酒店管理集团强关联资源}

国内酒店管理集团往往隶属于某个集团, 背靠集团的 巨大资源, 比如房地产、旅游景区、社区、航空、客户等。 例如华侨城酒店管理集团是华侨城集团的组成部分, 背靠 华侨城巨大的景区、社区、房地产资源和客户群体; 碧桂 园风凰国际酒店管理公司则背靠房地产开发商碧桂园集 团, 集团的巨大社区与业主群体是其酒店的重要资源; 首 旅酒店集团可以依托首旅集团的餐饮、旅行社、汽车服务 业务; 深航酒店集团、海航酒店集团则分别与深圳航空、 海南航空的航空业务存在着交集。下表是具有代表性的国 内酒店管理集团（公司）的母集团公司资源表:

表 1 部分酒店管理集团母集团公司资源表

\begin{tabular}{|c|c|c|}
\hline 序号 & 酒店管理集团 & 资源情况 \\
\hline 1 & 锦江国际 & $\begin{array}{l}\text { 酒店、旅游、客运物流、地产、物业、实业、 } \\
\text { 金融 }\end{array}$ \\
\hline 2 & 华侨城 & $\begin{array}{l}\text { 物业管理、商业区、主题公园、主题景区、 } \\
\text { Loft 创意文化园、艺术馆群、文化演艺、欢乐 } \\
\text { 谷、东部华侨城、世界之窗、旅游+地产模式 } \\
\text { 项目、康佳集团等 }\end{array}$ \\
\hline 3 & 维也纳酒店 & 美食宫餐饮连锁 \\
\hline 4 & 南京金陵 & $\begin{array}{l}\text { 房地产、生态旅游目的地、商务区、养老社区、 } \\
\text { 金陵贸易、糖酒食品公司 }\end{array}$ \\
\hline 5 & 首旅集团 & $\begin{array}{l}\text { 景区 (北京展览馆、高尔夫俱乐部、梨园剧场、 } \\
\text { 生态景区等)、旅行社、商业、餐饮、交通 } \\
\end{array}$ \\
\hline 6 & 碧桂园凤凰 & 房地产、装修、物业管理 \\
\hline 7 & 万达酒店 & $\begin{array}{l}\text { 商业地产 (万达广场)、连锁百货 (万达百货)、 } \\
\text { 文化旅游 (万达电影、万达大歌星、影视产业 } \\
\text { 园、主题公园、影视制作、电影发行、报刊传 } \\
\text { 媒) }\end{array}$ \\
\hline 8 & 海航集团 & 航空、候机楼、租车、免税品 \\
\hline 9 & 四川岷山 & 食品公司、建筑工程公司、装饰工程、旅行社 \\
\hline 10 & 山东银座 & $\begin{array}{l}\text { 汽车租赁、销售, 百货、超市、家居、网上商 } \\
\text { 城、旅游 }\end{array}$ \\
\hline
\end{tabular}

表中所列酒店管理集团 (公司) 包含的资源类型覆盖 较广, 其他未列的国内酒店管理集团几乎都有自己的丰富 的客户资源。事实上, 对国内近百家的酒店管理集团所属 集团公司资料进行分析整理后, 可以得出结论为了增加利 润点、分散风险而采取的多元化战略决定了母集团都拥有 其他产业、行业、公司的资源。即使业务构成纯粹的连锁 酒店集团也会在其主业上延伸餐饮、采购等业务, 有的则
采取与旅游目的地合作的方式拥有资源。酒店管理集团全 部或主要隶属于其母集团, 是母集团发展战略的重要组成 部分, 因此在获得和开发其他业务的客户数据与资源方面 具有先天优势。

\section{2 强关联资源的分析}

现代营销理论理论迎合互联网时代需求, 以用户的需 求为中心, 颠覆了传统的营销理论, 取得了巨大的成就。 近年来多手可热的互联网思维可以说就是创新迎合新经营 销售环境的俗称。除了实践, 在理论上也取得了很多成就, 例如 4C 理论聚焦于消费者需求 (Consumer wants and needs)、消费者愿意支付的成本 (Consumer's cost to satisfy that want or need)、消费者的便利性 (Convenience to buy)、 与消费者沟通 (Commuication); $4 \mathrm{R}$ 理论则立足于关联 (Relevant)、反应（Reaction）、关系（Relationship）、报酬 (Reward)。这些理论都得到了成功的印证, 具有一个共同 的特征, 即以客户为中心, 认为提供的产品、价格、使用 都是基于获取或创造用户需求的前提。

酒店管理集团 (公司) 的强关联资源表现出能开展互 联网营销的鲜明特点:

(1) 具有与客户直接连接的特点, 例如旅游目的地、 物业、房地产、商铺、旅行社, 用户在指定地点与集团业 务产生连接, 并获得用户数据。例如, 用户在购置房产后 入住, 日常生活中, “出门七件事, 柴米油盐酱醋茶”, 这 些都无法脱离与物业管理机构的直接接触。

（2）客户资料翔实，消费能力强。物业管理、房地产、 旅行社、景区等全部或部分业务需要用户登记资料, 从而 获得用户的第一手翔实全面的数据。一般而言, 这些用户 产生高额购买的行为较多, 具有较高的消费能力。

（3）客户数据与酒店住客数据具有交叉重合的特点。 酒店住客入住时需要登记个人信息, 因此存在着协同分析 住客在母集团其他行为活动数据的基础。尤其是高星级酒 店的住客与社区高档消费更存在着数据挖掘、个性化推荐 产品与服务的极大可能。

以创建业界 “旅游+地产” 模式的华侨城集团为例, 该 集团本部是位于深圳市南山区的华侨城区, 占地 4.8 平方 公里, 区内包括世界之窗、欢乐谷等景区资源，拥有华侨 城大酒店、威尼斯酒店、欢乐客栈等高档、连锁酒店。居 住与商业楼盘在其中错落分布, 业主 (租户) 的人口规模 达 20 万以上，该城区房地产产品价格高企，业主的平均消 费能力较强。物业管理机构通过了解与掌握业主资料以提 供更好的服务, 景区、酒店、商圈、医院、学校都能获取 不同深度与广度的用户数据。城区能提供丰富的服务资源, 希望能够在对的时间对的地点以对的形式推荐给对的人, 
这样在整个消费生态圈里具有产品、连接、需求与动力。

因此, 可以考虑把目前处于分散状态的用户数据、服 务资源进行整合, 提高用户的体验和销售产品, 达到 “双 赢” 的效果。酒店具有较好的信息化基础、直接接触市场、 产品标准化等特点, 较为适合作为资源整合的主体。

\section{3. 以酒店为主体的强关联资源整合}

\section{1 整合营销的传播规划模式}

根据舒尔茨等提出的整合营销传播规划模式 (如图 1 所示), 该模式将规划的焦点置于消费者及潜在消费者身 上, 将他们的行为信息作为市场细分的工具。依据该模式 共分为 8 个步骤: 建立消费者数据库 (包括人口统计资料、 心理统计资料、购买记录)、数据区别与分类 (以其行为细 分市场)、接触管理 (选择并决定营销者在什么时间地点状 态下与消费者沟通)、制定传播沟通战略、品牌战略、营销 目标、整合运用营销传播工具、选择有助于达成传播目标 的战术。

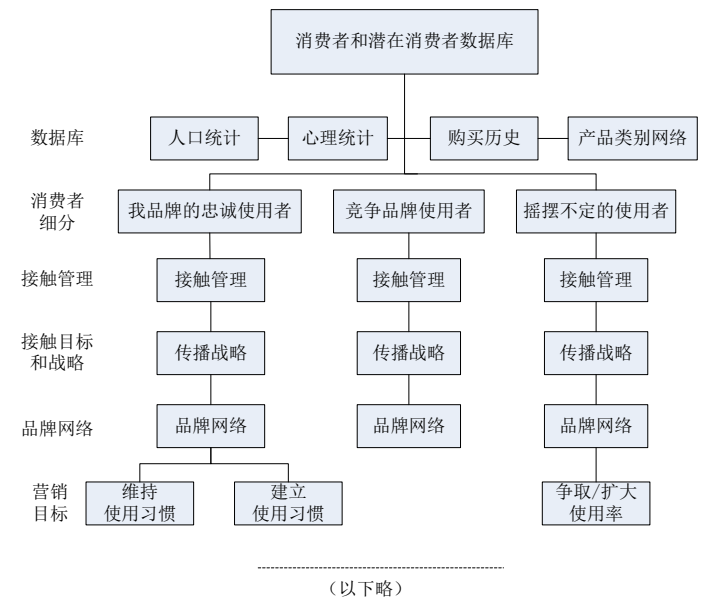

图 1 整合营销传播规划模式

在这个模式中, 要实现整合营销的最大门监就是消费 者数据库的建设, 这需要强大的与消费者接触的渠道, 其 获取成本非常昂贵。而酒店管理集团在这方面具有得天独 厚的优势, 依托母集团公司获取各种强关联资源的支持, 其中就有着核心的消费者数据。后期可以通过消费者细分、 接触管理等进行相应的营销, 从而提供了足够的操作空间。 但对于具体的酒店管理集团而言, 受其母集团的产权结构、 管理架构等制约, 在具体操作时很有可能陷入困境。

\section{2 以酒店管理集团为主体进行资源整合营销的制约因} 素分析

（1）管理体制制约。目前酒店管理集团的产权结构主
要分为独立型和依附母集团型。前者以华住酒店集团为代 表, 其绝对主营业务就是酒店, 因此以酒店为主体进行资 源整合可能遭受的压力较少; 后者则占绝大多数, 酒店管 理集团只是母集团的一部分, 且由于酒店业普遍亏损的现 状, 在母集团内部并没有很高的地位, 常不为高层领导 “待 见”, 因此整合受到的阻挠压力会比较大。一旦方案的前景 存在着不确定性时容易被更有影响力的其他子集团阻挠。

目前, 酒店管理集团在其总集团的组织架构中表现出 不同的治理结构, 包括事业部制独立运营、独立上市、事 业部制非独立运营等, 代表集团分别是万达集团、锦江国 际、华侨城集团。不同的治理结构在跨集团组织协调时表 现截然不同的特点。例如事业部制独立运营或独立上市结 构下酒店管理集团的独立性更强, 但与其他酒店资源的交 叉性相对较弱; 而事业部制非独立运营结构下酒店管理集 团公司的独立性不强, 其管理下的酒店分布在其他事业部 的经营范围, 一方面业务交叉较多关系较为密切, 容易达 成共识; 另一方面则没有强有力的管理效力, 受各方制约 较多, 无法保证方案顺利推行。

（2）产权结构的制约。如果酒店管理集团的产权是民 营的, 则机制往往比较灵活, 资源整合方案被通过的可能 性就增大; 如果是国有制的, 则由于国企的资源抢占型业 务导向和行政化特点有可能导致高层管理层不愿意承受风 险, 从而降低使用资源整合方案的可能性。

（3）企业文化与观念制约。进行资源的整合营销是一 个新的事物, 涉及到跨部门、新技术引进、新旧模式转换 以及盈利目标等风险, 部分保守型风格的管理层有可能抱 着 “多一事不如少一事” 的观念, 在当前经营状况尚佳且 没有重大的环境变化时只求守固, 不求创新。

（4）技术层面制约。技术层面包括整合的技术方法和 评估体系的建立。目前在一些领域上出现了资源整合的尝 试, 例如万科、花样年集团布局的社区 $\mathrm{O} 2 \mathrm{O}$, 但尚缺少成 熟的模式与方案, 都是在尝试的过程中不断摸索。希望在 包含更多商业资源的社区进行更大层面上的整合则更是缺 乏可操作的成熟的概念、流程、平台与方法。评估体系的 建立则是用于辅助管理层决策, 通过建立直接经济效益、 间接直接效益、联合效益等指标体系将整合营销的结果进 行量化。

\section{3 以酒店管理集团为主体的整合营销的形式}

整合营销的实质就是以消费者和潜在消费者为中心进 行精准营销，在互联网条件下，从资金筹集、酒店运营、 整合数据、联合营销以及任何环节上都可能实现整合营销。

第一阶段是酒店管理集团的内部整合, 创建连接点。 许多酒店管理集团辖下酒店形态各异, 档次不一样, 空间 
分布广泛, 管理体制复杂, 产权结构混乱, 因此其内部也 可能需要整合, 包括理顺管理体制、联合采购、统一服务 标准、内部资源共享等。酒店以实现线上线下网状连接为 目标, 把酒店的员工、住客、设施、场所、服务都当成是 一个连接点, 并可以无限辐射与其他点连接, 而核心就是 酒店住客;

第二阶段是确定酒店与强关联资源优先整合。将强关 联资源纳入酒店自身的关联网, 例如物业管理、景区等的 用户数据, 实现整合用户数据这个核心步骤。建立评估体 系, 尝试联动一体化考核机制, 不以一个部门的一得一失 来评估其绩效, 避免不同部门因自身利益消极对待整合甚 至阻挠。

第三阶段是可考虑与其他强关联资源的整合, 例如房 地产、旅行社等。甚至可以尝试集团外部资源的整合, 例 如资金投入可引入风险投资与民营资本等第三方资本，甚 至可以探索使用众筹的方式获得资金; 可与拥有用户大数 据的运营商、互联网企业合作，引进技术，共享数据等。

\section{4 以酒店管理集团为主体的整合营销的建议}

（1）自上而下原则。以酒店管理集团为主体进行整合 营销首先需要获得高层领导的支持。整合营销的实际是经 营模式的变化, 必然要涉及到人事与技术问题, 是一个典 型的 “一把手工程”。无数的信息化项目失败原因就是由于 得不到自上而下的足够力度的支持, 因此整合工程首先需 要高于酒店管理集团层面的管理人员负责决策、领导、协 调、控制, 并提供足够的资源。

（2）先易后难原则。在全面整合的条件不成熟时, 可 以考虑在一些易于整合的细处着眼, 以点带面, 形成影响 力, 最终在条件许可时再进行全面的整合。例如长隆欢乐 世界为长隆酒店的住客设置快速通道, 珠海长隆横琴湾酒 店的住客拥有优先进入海洋王国和入园专用通道等特权, 三亚亚龙湾酒店为住客建设通往沙滩的专用通道等, 这些 措施具有互联网思维的维形, 其核心是资源的整合, 在具 体实施时难度不大, 额外投入很少, 却在给住客节省时间 之余让住客的尊贵感油然而生, 从而产生更强的用户粘性。 当这些相对容易实行的整合措施产生影响力后就可以将其 纳入整体整合方案作为有力的支撑。

(3) 先内后外原则。对于部分酒店管理集团来说, 首 先是要提高自身内部的整合能力, 比如营销方法、品牌管 理、服务标准等都能体现出酒店整合战略的优势, 以酒店 为主战场, 尝试酒店在连接中的作用, 寻受传统服务之外 的增值服务形式。从互联网的视角来看, 酒店以消费者为 中心的联接网会不断滚动扩大, 可以容纳更多的其他网状 连接物。例如酒店可以与家具供应商合作, 不再采购家具,
而是允许供应商在酒店摆放其品牌家具, 住客若感兴趣可 以通过扫描家具上的二维码进行了解与购买。逐步实现每 一个空间每一个设备每一个工作人员都能与消费者直接相 连。

（4）重视技术，在实践中培养核心人员队伍。整合工 作最终还要落实到个人, 再好的方案与设想没有高效有力 的贯彻执行者最终都变成空谈。重视技术不光是重视软硬 件平台上的技术人员, 还要重视管理人员, 尤其是既懂技 术又懂管理, 具有大局观的管理人员。在实施方案的过程 中以实践带动人员水平的提高, 让其迅速融入技术与人的 交涉中。可以考虑使用原型法让相关人员先完成较为简单 的项目, 再在此项目基础上完善与提高, 从而让参与人员 快速地介入整个过程, 快速成长。

\section{4. 结语}

未来时代是整合的时代, 单独提供产品的商业模式将 会渐渐式微。国内酒店的竞争是很大的, 怎样才能更进一 步, 怎样才能不被新模式革命? 整合是必经之路。整合资 源意味着以消费者为中心, 适应未来互联网时代人群的消 费方式和趋向。谁先占有用户, 谁将赢得这场看不见硝烟 的战争。

\section{参考文献(References)}

[1] China Tourist Hotels Association, The 2013 report of China hotel management company (group) development, Beijing, 2014.

[2] ZHU Chengqiang and LV Lili, "Research on Relationship between International Hotel Management Groups and Chinese Property Owners under Management Contract”,Tourism Science, vol. 25, no. 3, pp.67-74,2011.

[3] http://www.jinjiang.com/

[4] http://www.chinaoct.com

[5] http://www.wyn88.com/

[6] http://www.jinlinghotel.com/Chinese/JYFZ/JYFZ-JLFD.aspx

[7] http://www.btg.com.cn/business/

[8] Lauterborn, B., “New Marketing Litany: Four P's Passe: C-Words Take Over,” Advertising Age, vol.61, no. 41, p. 26.

[9] Elliott Ettenberg, The Next Economy: Will You Know Where Your Customers Are? .New York, McGraw-Hill, 2001.

[10] Don E. Schultz, Stanley I. Tannenbaum, Robert F. Lauterborn, The New Marketing Paradigm: Integrated Marketing Communications .New York, McGraw-Hill, 1996.

[11] Stephen P.Robbins, Management(7th version), Beijing, China Renmin University Press, 2004. 